\title{
Comparing Properties of Rising Factorial Function with Properties of Falling Factorial Function
}

\author{
Weida Qin and Wusheng Wang \\ School of Mathematics and Statistics, Hechi University \\ Guangxi, Yizhou 546300, P. R. China \\ wang4896@126.com
}

Keywords: Falling factorial function; Rising factorial function; Comparing; Property 2000 MSC 26D10, 26D15, 26D20, 45A99

\begin{abstract}
This paper firstly introduces the definition of the falling factorial polynomial and the definition of the rising factorial polynomial, and then proves their properties by analysis methods, finally concludes similar properties of rising factorial function with falling factorial function and different properties of rising factorial function with falling factorial function.
\end{abstract}

\section{Introduction}

The factorial operation is encountered in many areas of mathematics, notably in combinatorics, algebra, and mathematical analysis. The falling factorial polynomial (sometimes called the descending factorial, falling sequential product, lower factorial) is defined:

$$
x^{(n)}=\prod_{j=0}^{n-1}(x-y), x \in R, n \in N .
$$

Remark 1. From the definition of the falling factorial polynomial, we see that $x^{(0)}=1$; $x^{(1)}=x ; x^{(2)}=x(x-1) ;$ and $x^{(n)}=0 ;$ when $x-n \in\{\cdots,-2,-1\} ;$ and we have

$$
x^{(n)}=\prod_{j=0}^{n-1}(x-j)=\frac{\Gamma(x+1)}{\Gamma(x+1-n)}=n !\left(\begin{array}{l}
x \\
n
\end{array}\right), n \in N, x-n \in R \backslash\{\cdots,-2,-1\}
$$

Where $\Gamma$ denotes the special gamma function.

The rising factorial polynomial (sometimes called the Pochhammer function, Pochhammer polynomial, ascending factorial, rising sequential product, upper factorial) is defined

$$
x^{\bar{n}}=\prod_{j=0}^{n-1}(x+j), x \in R, n \in N \text {. }
$$

Remark 2. From the definition of the rising factorial polynomial, we have $x^{\overline{0}}=1 ; x^{\overline{1}}=x$ and $x^{\overline{2}}=x(x+1)$, and $t^{\bar{n}}=0$ when $x \in\{\cdots,-2,-1\}$; and we have

$$
x^{\bar{n}}=\prod_{j=0}^{n-1}(x+j)=\frac{\Gamma(x+n)}{\Gamma(x)}=n !\left(\begin{array}{c}
x+n-1 \\
n
\end{array}\right), x \in R \backslash\{\cdots,-2,-1,0\}, n \in N .
$$

Remark 3. From the definitions of the falling and rising factorial polynomials, we have

$$
x^{\bar{n}}=(x+n-1)^{(n)}=(-1)^{n}(-x)^{(n)} \text {. }
$$

\section{Preliminary Definitions and Properties}

Extending the two above definitions from an integer $\mathrm{n}$ to an arbitrary real number $\mathrm{y}$, the power 
function is defined by $[3,4,6]$.

$$
\begin{aligned}
& x^{(y)}=\frac{\Gamma(x+1)}{\Gamma(x+1-y)}=\left(\begin{array}{c}
x+n-1 \\
n
\end{array}\right), \text { for }, x \in R, x-y \in R, \backslash\{\cdots,-2,-1,\}, \\
& x^{\bar{y}}=\frac{\Gamma(x+y)}{\Gamma(x)}, \text { for }, y \in R, x \in R \backslash\{\cdots,-2,-1,0\}
\end{aligned}
$$

We assume that $x^{(y)}=0$ when $x-y \in\{\cdots,-2,-1\}$; and $0^{\bar{y}}=0, x^{\bar{y}}=0$ when $x \in\{\cdots,-2,-1\}$.

Remark 4. Using the properties of the Gamma function it is easily seen that $x^{(y)}>0$ when $x>-1, x-y>-1$, and $x^{\bar{y}}>-1$, when $x>0, x+y>0$,

We will list some of the properties of the falling factorial function with their proofs.

Lemma 1. ([3], Theorem 2.1.). Assume that the following factorial functions are well defined. $\Delta x^{(y)}=y x^{(y-1)}$,

$\Delta^{k} x^{(y)}=\frac{\Gamma(y+1)}{\Gamma(y+1-k)} x^{(y-k)}$

$(x-y) x^{(y)}=x^{(y+1)}$,

$x^{(x)}=\Gamma(x+1)$,

$x^{(y)} \leq r^{(y)}, x \leq r, y<x+1$,

$x^{(y z)} \geq\left(x^{(y)}\right)^{z}, 0<z<1$,

$x^{(y+z)}=(x-z)^{(y)} x^{(z)}$,

where $\Delta y(t)=y(t+1)-y(t)$.

Proof. The proof of (8). From (6), we have

$$
\begin{aligned}
& \Delta x^{(y)}=\frac{\Gamma(x+2)}{\Gamma(x+2-y)}-\frac{\Gamma(x+1)}{\Gamma(x+1-y)} \\
& =\frac{(x+1) \Gamma(x+1)}{\Gamma(x+1-(y-1))}-\frac{\Gamma(x+1-y) \Gamma(x+1)}{\Gamma(x+1-(y-1))} \\
& =y \frac{\Gamma(x+1)}{\Gamma(x+1-(y-1))}=y x^{(y-1)} .
\end{aligned}
$$

The proof of (9). From (8), we get

$$
\Delta^{k} x^{(y)}=\Delta^{k-1}\left(\Delta x^{(y)}\right)=\Delta^{k-1} y x^{(y-1)}=\frac{\Gamma(y+1)}{\Gamma(y+1-k)} x^{(y-k)} \text {. }
$$

The proof of (10). From (6), we have 


$$
x^{(y+1)}=\frac{\Gamma(x+1)}{\Gamma(x+1-(y-1))}=\frac{(x-y) \Gamma(x+1)}{\Gamma(x+1-y)}=(x-y) x^{(y)} .
$$

The proof of (11). From (6), we obtain

$$
x^{(x)}=\frac{\Gamma(x+1)}{\Gamma(x+1-x)}=\Gamma(x+1) \text {. }
$$

The proof of (12). By Euler's infinite product.

$$
\Gamma(x)=\frac{1}{x} \prod_{n=1}^{\infty} \frac{\left(1+\frac{1}{n}\right)^{x}}{1+\frac{x}{n}} .
$$

For $x \leq r, y<x+1$, we have

$$
\begin{aligned}
& x^{(y)}=\frac{\Gamma(x+1)}{\Gamma(x+1-y)}=\frac{x+1-y}{x+1} \prod_{n=1}^{\infty} \frac{\left(1+\frac{1}{n}\right)^{x+1}}{1+\frac{x+1}{n}} \frac{1+\frac{x+1-y}{n}}{\left(1+\frac{1}{n}\right)^{x+1-y}} \\
& =\frac{x+1-y}{x+1} \prod_{n=1}^{\infty} \frac{\left(1+\frac{1}{n}\right)^{y}(x+1-y+n)}{x+1+n} \\
& =\left(1-\frac{y}{x+1}\right) \prod_{n=1}^{\infty}\left(1+\frac{1}{n}\right)^{y}\left(1-\frac{y}{x+1+n}\right) \\
& \leq\left(1-\frac{y}{r+1}\right) \prod_{n=1}^{\infty}\left(1+\frac{1}{n}\right)^{y}\left(1-\frac{y}{r+1+n}\right) \\
& =r^{(y)} .
\end{aligned}
$$

The proof of (13). From the log-convexity property of the gamma function.

$$
\Gamma(z a+(1-z) b) \leq(\Gamma(a))^{z}(\Gamma(b))^{1-z}, 0<z<1,
$$

We obtain

$$
\begin{aligned}
& x^{(y z)}=\frac{\Gamma(x+1)}{\Gamma(x+1-y z)}=\frac{\Gamma(x+1)}{\Gamma(z(x+1-y)+(1-z)(x+1))} \\
& \geq \frac{\Gamma(x+1)}{(\Gamma(x+1-y))^{z}(\Gamma(x+1))^{1-z}}=\left(x^{(y)}\right)^{z} .
\end{aligned}
$$

The proof of (14). From (6), we have 


$$
\begin{aligned}
& x^{(y+z)}=\frac{\Gamma(x+1)}{\Gamma(x-z+1-y)} \frac{\Gamma(x-z+1)}{\Gamma(x+1-z)} \\
& =\frac{\Gamma(x-z+1)}{\Gamma(x-z+1-y)} \frac{\Gamma(x+1)}{\Gamma(x+1-z)}=(x-z)^{(y)} x^{(z)} .
\end{aligned}
$$

These complete the proofs.

\section{Main Results}

We will list some of the properties of the rising factorial function with their proofs.

Theorem 1. ([3], Theorem 2.1.). Assume that the following factorial functions are well defined.

$$
\begin{aligned}
& \nabla x^{\bar{y}}=y x^{\overline{y-1}} ; \\
& \nabla^{k} x^{\bar{y}}=\frac{\Gamma(y+1)}{\Gamma(y+1-k)} x^{\overline{y-k}} \\
& (x+y) x^{\bar{y}}=x^{\overline{y+1}} \\
& x^{\bar{x}}=\frac{\Gamma(2 x)}{\Gamma(x)} \\
& x^{\bar{y}} \geq r^{\bar{y}}, x \leq r, y<x, \\
& x^{\overline{y z}} \leq\left(x^{\bar{y}}\right)^{z}, 0<y<1, \\
& x^{\overline{y+z}}=(x+z)^{\bar{y}} x^{\bar{z}} ;
\end{aligned}
$$

Where $\nabla x(t)=x(t)-x(t-1)$.

Proof. The proof of (24)

$$
\begin{aligned}
& \nabla x^{\bar{y}}=x^{\bar{y}}-(x-1)^{\bar{y}}=\frac{\Gamma(x+y)}{\Gamma(x)}-\frac{\Gamma(x+y-1)}{\Gamma(x-1)} \\
& =\frac{(x+y-1) \Gamma(x+y-1)}{\Gamma(x)}-\frac{(x-1) \Gamma(x+y-1)}{\Gamma(x)} \\
& =y \frac{\Gamma(x+y-1)}{\Gamma(x)}=y x^{\overline{y-1}} .
\end{aligned}
$$

This completes the proof.

The proof of (25)

$$
\nabla^{k} x^{\bar{y}}=\nabla^{k-1} \nabla x^{\bar{y}}=y \nabla^{k-1} x^{\overline{y-1}}=y(y-1) \square(y-k+1) x^{\overline{y-k}}=\frac{\Gamma(y+1)}{\Gamma(y+1-k)} x^{\overline{y-k}} \text {. }
$$


This completes the proof.

The proof of (26). From (7), we have

$$
x^{\overline{y+1}}=\frac{\Gamma(x+y+1)}{\Gamma(x)}=(x+y) \frac{\Gamma(x+y)}{\Gamma(x)}=(x+y) x^{\bar{y}} .
$$

This completes the proof.

The proof of (27). From (7), we have

$$
x^{\bar{x}}=\frac{\Gamma(2 x)}{\Gamma(x)} \text {. }
$$

This completes the proof.

The proof of (28). By Euler's infinite product.

$$
\Gamma(x)=\frac{1}{x} \prod_{n=1}^{\infty} \frac{\left(1+\frac{1}{n}\right)^{x}}{1+\frac{x}{n}} .
$$

For $x \leq r, y<x$, we have

$$
\begin{aligned}
& x^{\bar{y}}=\frac{\Gamma(x+y)}{\Gamma(x)}=\frac{x}{x+y} \prod_{n=1}^{\infty} \frac{\left(1+\frac{1}{n}\right)^{x+y}}{1+\frac{x+y}{n}} \frac{1+\frac{x}{n}}{\left(1+\frac{1}{n}\right)^{x}} \\
& =\frac{x}{x+y} \prod_{n=1}^{\infty} \frac{\left(1+\frac{1}{n}\right)^{y}(x+n)}{x+y+n}=\frac{1}{1+\frac{y}{x}} \prod_{n=1}^{\infty} \frac{\left(1+\frac{1}{n}\right)^{y}}{1-\frac{y}{x+n}} \\
& =\frac{1}{1-\frac{y}{x}} \prod_{n=1}^{\infty} \frac{\left(1-\frac{1}{n}\right)^{y}}{x+n} \\
& \geq \frac{1}{1-\frac{y}{r}} \prod_{n=1}^{\infty} \frac{\left(1-\frac{1}{n}\right)^{y}}{r+n} \\
& =r^{\bar{y}}
\end{aligned}
$$

This completes the proof.

The proof of (29). From the log-convexity property of the gamma function.

$$
\Gamma(z a+(1-z) b) \leq(\Gamma(a))^{z}(\Gamma(b))^{1-z}, 0<z<1,
$$

We obtain 


$$
\begin{aligned}
& x^{\overline{y z}}=\frac{\Gamma(x+y z)}{\Gamma(x)}=\frac{\Gamma(z(x+y)+(1-z) x)}{\Gamma(x)} \\
& \leq \frac{(\Gamma(x+y))^{z}(\Gamma(x))^{1-z}}{\Gamma(x)}=\left(x^{y}\right)^{z} .
\end{aligned}
$$

The proof of (30). From (7), we have

$$
\begin{aligned}
& x^{\overline{y+z}}=\frac{\Gamma(x+y+z)}{\Gamma(x)} \frac{\Gamma(x+z)}{\Gamma(x+z)} \\
& =\frac{\Gamma(x+z+y)}{\Gamma(x+z)} \frac{\Gamma(x+z)}{\Gamma(x)}=(x+z)^{\bar{y}} x^{\bar{z}} .
\end{aligned}
$$

These complete the proofs.

\section{Conclusion}

Similar properties of rising factorial function with falling factorial function.

$$
\begin{aligned}
& \Delta x^{(y)}=y x^{(y-1)}, \nabla x^{\bar{y}}=y x^{\overline{y-1}} \\
& \Delta^{k} x^{(y)}=\frac{\Gamma(y+1)}{\Gamma(y+1-k)} x^{(y-k)}, \nabla^{k} x^{\bar{y}}=\frac{\Gamma(y+1)}{\Gamma(y+1-k)} x^{\overline{y-k}} .
\end{aligned}
$$

Different properties of rising factorial function with falling factorial function $(x-y) x^{(y)}=x^{(y+1)},(x+y) x^{\bar{y}}=x^{\overline{y+1}}$;

$$
\begin{aligned}
& x^{(x)}=\Gamma(x+1), x^{\bar{x}}=\frac{\Gamma(2 x)}{\Gamma(x)} ; \\
& x^{(y)} \leq r^{(y)}, x^{\bar{y}} \geq r^{\bar{y}}, x \leq r, y<x ; \\
& x^{(y z)} \geq\left(r^{(y)}\right)^{z}, x^{\overline{y z}} \leq\left(x^{\bar{y}}\right)^{z}, 0<z<1 ; \\
& x^{(y+z)}=(x-z)^{(y)} x^{(z)}, x^{\overline{y+z}}=(x+z)^{\bar{y}} x^{\bar{z}} .
\end{aligned}
$$

\section{Acknowledgments}

This research was supported by National Natural Science Foundation of China (Project No.11561019, 11161018) and Natural Science Foundation of Guangxi Autonomous Region of China (No. 2016GXNSFAA380090) and Hechi Universiry master's degree awarded in 2016 to build the project fund(No.2016YT003).

\section{References}

[1] Podlubny, Fractional Differential Equations, Academic Press, New York, 1999. 
[2] G Jumarie,Fractional partial differential equations and modified Riemann-Liouville derivative new methods for solution, Journal of Applied Mathematics and Computing, 2007,24(1):31-48.

[3] F. M. Atici and P. W. Eloe, A transform method in discrete fractional calculus, Int. J. Difference Equ., 2007, 2(2): 165-176.

[4] G.A., Anastassiou, Nabla discrete fractional calculus and nabla inequalities, Math. Comput . Modelling, 2010, 51(5-6):562-571.

[5] Holm, M.T.: The Theory of discrete fractional calculus: development and application. $\mathrm{PhD}$ thesis, University of Nebraska-Lincoln, Lincoln, Nebraska (2011)

[6] R. A. C. Ferreira, A discrete fractional Gronwall inequality, Proc. Amer.Math. Soc., 2012, 140: 1605-1612.

[7] F Chen, Y Zhou, Existence and Ulam Stability of Solutions for Discrete Fractional Boundary Value Problem, Discrete Dynamics in Nature \& Society, 2013,2013, Article ID 459161, 1-7.

[8] Y Chen, X Tang, The difference between a class of discrete fractional and integer order boundary value problems, Communications in Nonlinear Science \& Numerica, 2014,19(12):4057-4067.

[9] J.J Mohan, GVSR Deekshitulu, Solutions of Nabla Fractional Difference Equations Using N-Transforms, Communications in Mathematics and Statistics, 2014, 2(1):1-16.

[10]Q.H Feng, Some new generalized GronwallCBellman type discrete fractional inequalities, Applied Mathematics and Computation, 2015, 259: 403-411.

[11]H.D Liu and F.W Meng, Some new generalized Volterra-Fredholm type discrete fractional sum inequalities and their applications, Journal of Inequalities and Applications, 2016, 2016:213. $1-16$.

[12] Samet Erden, Mehmet Zeki Sarikaya, Generalized Pompeiu type inequalities for local fractional integrals and its applications, Applied Mathematics and Computation, 2016, 274(1): 282-291.

[13]L Erbe, CS Goodrich, B Jia, A Peterson, Survey of the qualitative properties of fractional difference operators: monotonicity, convexity, and asymptotic behavior of solutions, Advances in Difference Equations, 2016, 2016(1):1-31. 